Kulhara, P. \& Chadda, R. (1987) Study of negative symptoms in schizophrenia and depression. Comprehensive Psychiatry, 28, 229-235.

de LeON, J., WILsON, W. H. \& Simpson, G. M. (1989) Measurement of negative symptoms in schizophrenia. Psychiatric Development 3, 211-234.

SING LeE

Prince of Wales Hospital

HELEN LEUNG

Shatin, $N T$

Hong Kong

\section{Hospital Anxiety and Depression Scale}

SIR: Chaturvedi (Journal, August 1991, 159, 298) claims that in his clinical study the Hospital Anxiety and Depression Scale (HAD Scale) could 'hardly discriminate' between anxiety and depression, and recommends that the two subscales be combined into a single score. Chaturvedi does not state whether he was using the HAD Scale in English in his Indian subjects, or a translated version; nor whether he evaluated the linguistic, conceptual or scale equivalence (Marsella, 1987) of the HAD Scale in the Indian setting.

With colleagues in Lahore (Pakistan), I have considerable experience with the HAD Scale both in English and in our Urdu version (Mumford et al, $1991 a$ ). The Urdu version is to be preferred unless the subject is very fluent in English: people generally express their emotional state better in their mothertongue. In one study, medical out-patients in Lahore completed the HAD Scale in Urdu, and DSM-III-R diagnoses were made independently at interview (Mumford et al, 1991b). Among 14 patients with depressive disorders, the mean HAD Scale anxiety score was 12.9 and HAD Scale depression score was 9.4; among 17 patients with anxiety and panic disorders the scores were 11.2 and 6.9; and among 23 patients with no psychiatric diagnosis they were 6.8 and 4.9 respectively.

These results are consistent with Chaturvedi's findings, but not with his conclusions. The HAD Scale depression (anhedonia) subscores were highest in the depressed patients, the most important clinical group to screen for; after excluding these patients, the HAD Scale anxiety subscores then successfully identified the anxiety and panic disorders. (High levels of anxiety among patients with depressive disorders is a common clinical finding.) In the whole sample, the HAD Scale anxiety items consistently correlated more highly with the anxiety subscore than they did with the depression subscore; and vice versa with the HAD Scale depression items.
Admittedly, the two subscales of the HAD Scale are not similarly calibrated and may require different cut-off scores for maximum screening efficiency. The fact that the authors have given two cut-off points (for possible and probable clinical significance) underlines the arbitrariness of any threshold when traits are continuously distributed in a population. However using the 10/11 cut-off on both the anxiety and the depression subscales, we found that the HAD Scale identified patients with DSM-III-R disorders in Lahore medical clinics with a sensitivity of $83 \%$ and specificity of $78 \%$.

The HAD Scale does not discriminate perfectly between anxiety and depression partly because it only taps the subjective component of the mood disorders. The HAD Scale was designed as a brief screening instrument for medical patients with concurrent physical illness. It is not a comprehensive inventory of anxiety or depressive symptoms and signs; it should not be used alone to make a clinical diagnosis, but only to indicate the probability of a psychiatric disorder being present. In my view, the HAD Scale fulfils this function satisfactorily both in Britain and in Pakistan.

Marsella, A. J. (1987) The measurement of depressive experience and disorder across cultures. In The Measurement of Depression (ed. A. J. Marsella). New York: Guilford Press.

MUMford, D. B., TAREen, I. A. K., BAJWA, M. A. Z., el al (1991a) The translation and evaluation of an Urdu version of the Hospital Anxiety and Depression Scale. Acta Psychiatrica Scandinavica, 83, 81-85.

— - - BнАтT, M. R., et al (1991b) An investigation of 'functional' somatic symptoms among patients attending hospital medical clinics in Pakistan: II. Using somatic symptoms to identify patients with psychiatric disorders. Journal of Psychosomatic Research, 35, 257-264.

Department of Psychiatry

DAvid B. Mumford

University of Leeds

25 Hyde Terrace

Leeds LS2 9LT

\section{Neuroleptic Malignant Syndrome}

SIR: We read with interest White \& Robins' reply to our letter (Journal, June 1991, 158, 858-859), Dalkin \& Kennedy's views on catatonia and neuroleptic malignant syndrome (NMS) (Journal, June 1991, $158,859)$ and Otani $e t$ al's "Is the predisposition to neuroleptic malignant syndrome genetically transmitted?" (Journal, June 1991, 158, 850-853).

Dr Otani's cases illustrate the diagnostic dilemma that Drs White \& Robins expressed about catatonia and NMS. In the three cases of a mother and her two daughters who were diagnosed to have NMS, the mother had an episode while not on neuroleptics and 
another episode in which her catatonia and fever improved with ECT. The younger daughter also had an episode of fever and autonomic instability without rigidity with no neuroleptics. (Castillo suggested that muscle rigidity may be absent in lethal catatonia). These cases could be diagnosed as lethal catatonia rather than NMS.

Drs Dalkin \& Kennedy, and Drs White \& Robins, stated that the two conditions are indistinguishable on clinical grounds and the pathophysiologies are similar, although others (Castillo et al, 1989; Fleischhacker et al, 1990) attempted to differentiate between the two conditions. Fleischhacker believes that many patients, having been started on neuroleptics, diagnosed as NMS, might actually have been lethal catatonia. Is it possible that we are diagnosing the same condition that was first described by Calmeil in 1832 and numerous times thereafter as NMS when neuroleptics are prescribed, and as lethal catatonia when they are not?

Drs Otani et al mentioned in their paper that they could not find any reports on familial NMS in the literature. Stauder, in his original description of lethal catatonia, reported a family history of catatonia, including lethal catatonia, in half of his 27 cases.

Castillo, E., Rubin, R. \& Holsber Traschler, E. (1989) Clinical differentiation between lethal catatonia and malignant neuroleptic syndrome. American Journal of Psychiatry, 146, 324-328.

FLeischHACKER, W. W., UNTERWENGER, B., KANE, J. M., et al (1990) The neuroleptic syndrome and its differentiation from lethal catatonia. Acta Psychiatrica Scandinavica, 81, 3-5.

Stauder, K. H. (1870) Die todliche katatonie. Archiv fur Psychiatrie und Nervenkrankheiten, 102, 614-634.

T. K.S. TAN

Department of Psychological Medicine

National University Hospital

Singapore

St Mary Abbots Hospital

S. H. ONG

London W8 $5 L Q$

\section{Psychiatrists and citizens}

SIR: Dr Jim Birley's Presidential Address of July 1990, reproduced in the Journal (July 1991, 159, 1-6) is elegant, erudite, eloquent and timely.

At the first William Sargant Lecture, in July 1991, in the presence of Dr Sargant's widow, Dr Birley, in giving the vote of thanks to the speaker, chose to recall, as risible, Dr Sargant's objection to the Modecate study as unethical (Hirsch et al, 1973). This was a study carried out at the Institute of which Dr Birley was dean. The consent of the patients was neither sought nor obtained, the families were not informed and the general practitioners not asked to approve. Sixty-six per cent of the patients on placebo relapsed.

What makes Dr Sargant's observance of the "God given condition of eternal vigilance" a matter for levity, if not scorn? Does not Dr Sargant's action more appropriately "Deserve the love and thanks of man and woman"? (Paine, 1778).

Is there a price to be paid for thinking that 'eternal vigilance' begins at home?

Hirsch, S. R., Gaind, R., Rohde, P. D., et al (1973) Outpatient maintenance of chronic schizophrenic patients with long acting fluphenazine: double-blind placebo trial. British Medical Journal. $i, 633-637$.

Paine, T. (1778) Crisis.

12 Castle Garden

Gerald Samuel

Petersfield

Hampshire GU32 3AG

\section{Frégoli transformed}

SIR: We read with interest the report by O'Sullivan \& Dean (Journal, August 1991, 159, 274-277) of four cases of the Frégoli delusion occurring in puerperal illnesses. Previous references in the literature to delusional misidentification in this setting mentioned only the Capgras type (e.g. De Leo et al, 1985).

However, the descriptions of the patients, as reported, do not correspond to the classic one of Courbon \& Fail. The hallmark of the Frégoli phenomenon is the delusional misidentification of familiar persons disguised as others. By our reading, all four cases could have exhibited the phenomenologically related, but much rarer, intermetamorphosis delusion, which refers to the conviction that a key person has been physically transformed into another. Of course, patients often show an admixture of symptoms, which may make the exact delineation of phenomenology something of an exercise in sophistry.

Over the last seven decades, a plethora of mutually incompatible, psychodynamic explanations, reflecting the imagination and conceptual frameworks of individual authors, have been invoked to account for the Capgras and other delusions of misidentification. However, as Guze (1988) has succinctly pointed out, the psychotherapeutic process is intrinsically incapable of establishing the causal basis of psychopathology, and aetiological hypotheses generated during therapy are therefore of doubtful validity unless tested critically outside this situation. Of course, this does not imply that psychosocial factors do not play a part in the development of the content of such delusions - what remains to be proven is that such factors are necessary and sufficient. 